Physics

Physics Research Publications

\title{
Magnus force in discrete and continuous two-dimensional superfluids
}

\author{
Z. Gecse \\ S. Khlebnikov
}

This paper is posted at Purdue e-Pubs.

http://docs.lib.purdue.edu/physics_articles/158 


\title{
Magnus force in discrete and continuous two-dimensional superfluids
}

\author{
Z. Gecse and S. Khlebnikov \\ Department of Physics, Purdue University, West Lafayette, Indiana 47907, USA
}

(Received 31 March 2005; published 29 August 2005)

\begin{abstract}
Motion of vortices in two-dimensional superfluids in the classical limit is studied by solving the GrossPitaevskii equation numerically on a uniform lattice. We find that, in the presence of a superflow directed along one of the main lattice periods, vortices move with the superflow on fine lattices but perpendicular to it on coarse ones. We interpret this result as a transition from the full Magnus force in a Galilean-invariant limit to vanishing effective Magnus force in a discrete system, in agreement with the existing experiments on vortex motion in Josephson junction arrays.
\end{abstract}

DOI: 10.1103/PhysRevB.72.054525

PACS number(s): 74.81.Fa, 03.75.Lm

\section{INTRODUCTION}

There is a long-standing interest in how the Magnus force, acting on vortices in superfluids and superconductors, changes as the system moves away from the Galileaninvariant (GI) limit. Indeed, experiments indicate that the effective Magnus force is very small both in conventional bulk superconductors-except for very clean ones ${ }^{1}$ - and in "discrete" superconductors, formed by Josephson-junction arrays (JJAs). ${ }^{2}$ In the first instance, there is a convincing explanation for this smallness, based on the spectral flow of fermions at the vortex core. ${ }^{3-7}$ The spectral flow creates an additional force on the vortex that reduces the total, effective Magnus force nearly to zero. However, the second case has remained something of a mystery.

Various explanations of the smallness of the Magnus force in JJA have been reviewed in Ref. 8. One proposal is that in this case the Magnus force is proportional not to the total density of electrons, but only to the "offset charges," given by the deviation of the system from electrical neutrality. ${ }^{9}$ Another proposal is that the effective Magnus force vanishes exactly as a consequence of the particle-hole symmetry. ${ }^{10}$ However, Volovik ${ }^{8}$ has argued that the particle-hole symmetry in these systems is not exact, and as a result the effective Magnus force is nonzero, although small. Finally, we mention that when the Josephson barrier is metallic, cancellation of the Magnus force can be explained ${ }^{11}$ by a spectral flow mechanism similar to that in bulk superconductors. This, however, does not work when the barrier is insulating.

There is a common theme to the above proposals: they all make use of specific properties of the electronic spectra or, alternatively, of the particle-hole symmetry already at the level of an effective description-in terms of phases and charges of superconducting islands. ${ }^{10}$ As we will see below, that symmetry, present in the simplest model of JJA, results in fact from neglecting the coupling between phase gradients and density fluctuations. The question then is whether this assumption indeed applies in the discrete limit, or a nonzero Magnus force persists no matter how discrete the system becomes.

In this paper, we report results of a numerical study of the Magnus force. These results have been obtained by numerical solution of the classical Gross-Pitaevskii (GP) equation in two dimensions (2D). Since the classical approximation neglects the commutator of the Bose fields $\Psi, \Psi^{\dagger}$ in comparison with the average density $n_{\mathrm{ave}}=\left\langle\Psi^{\dagger} \Psi\right\rangle$, it requires that the number of particles per site be large enough. More precisely, the classical limit in JJA is reached when the Josephson energy is much larger than the charging energy. ${ }^{2}$ An equivalent condition is

$$
n_{\mathrm{ave}} a^{2} \gg \frac{1}{n_{\mathrm{ave}} \xi^{2}},
$$

where $a$ is the lattice spacing (we assume a square or a nearly square lattice) and $\xi$ is the "healing" length, defined below. The right-hand side of (1) is a dimensionless measure of the interaction strength.

To explore the role of discreteness, we solve the timedependent GP equation on uniform spatial lattices with different values of the lattice spacing $a$. The relevant length scale to which $a$ can be compared is the "healing" length $\xi$. When $a \lesssim \xi$, we reach the nearly GI limit, in which the GP equation describes a quasi-continuous neutral superfluid. Vortices have a core of size $\xi$, which is resolved by the lattice. In the opposite limit, $a \gg \xi$, vortices have no core, in the sense that there is no significant depletion of density anywhere. The lattice sites can then be thought of as corresponding to individual islands, each of which is characterized by a value of the phase variable - a model of a JJA.

More precisely, the Lagrangian of our model in rescaled variables, for the case of a square lattice, is

$$
L=\sum_{j} \psi_{j}^{\dagger}\left(i \partial_{t}-\frac{1}{2}\left|\psi_{j}\right|^{2}+\mathrm{const}\right) \psi_{j}-\frac{1}{a^{2}} \sum_{(i j)}\left|\psi_{i}-\psi_{j}\right|^{2},
$$

where the first sum is over all lattice sites, and the second is over all nearest neighbors. Note that if we write $\psi$ $=\sqrt{n} \exp (i \theta)$ and neglect fluctuation of density $\delta n=n-n_{\text {ave }}$ in the second (gradient) sum in Eq. (2), the classical equations of motion become invariant under the transformation $\theta \rightarrow$ $-\theta, \delta n \rightarrow-\delta n$. This is the particle-hole symmetry that was used in Ref. 10 to argue the absence of Magnus force in JJAs. Here, we study the complete Lagrangian (2), including the coupling between $\delta n$ and the phase gradients.

On a coarse lattice, and in the presence of superflow, the rotational invariance is broken so strongly that definition of the Magnus force becomes a nontrivial matter. Indeed, we 
have found that in general vortices and antivortices do not even move symmetrically with respect to the superflow. However, when the superflow is along one of the lattice's main periods, they do, and we concentrate on this case in what follows.

We have found that while on fine lattices vortices move with the superflow, as expected in the GI limit, on coarse lattices they move perpendicular to it. We interpret this as vanishing of the effective Magnus force in the discrete limit, in agreement with the experiments on JJAs. ${ }^{2}$

The paper is organized as follows. We describe details of the numerical procedure in Sec. II. In Sec. III, we describe a phenomenological model that we use to interpret our numerical results. This model allows us to convert measurements of the longitudinal and transverse velocities of a vortex into values of the effective Magnus and drag force coefficients. Numerical results are presented in Sec. IV. Section V is a conclusion.

\section{NUMERICAL METHOD}

\section{A. Dimensionless variables}

The continuum GP equation has the form

$$
i \frac{\partial \Psi}{\partial t}=-\frac{1}{2 m} \nabla^{2} \Psi+g|\Psi|^{2} \Psi,
$$

where $\Psi$ is a complex scalar, the order parameter of the superfluid, $m$ is the mass of a fluid particle (in units where $\hbar=1$ ), and $g$ is the interaction constant. For computational purposes, it is convenient to scale out the parameters by expressing length, time, and the order parameter in their "natural" units. A natural unit of length is the "healing" length

$$
\xi=\left(2 m g n_{\text {ave }}\right)^{-1 / 2},
$$

where $n_{\text {ave }}=\left\langle\Psi^{\dagger} \Psi\right\rangle$ is the average density of the fluid. Then, new, tilded variables are defined by the following relations:

$$
\begin{gathered}
x=\tilde{x} \xi, \\
t=\tilde{t} \frac{1}{g n_{\mathrm{ave}}}, \\
\Psi=\psi \sqrt{n_{\mathrm{ave}}},
\end{gathered}
$$

In the new variables, the GP equation simplifies to

$$
i \frac{\partial \psi}{\partial \widetilde{t}}=-\widetilde{\nabla}^{2} \psi+|\psi|^{2} \psi
$$

Note that by virtue of (7) the rescaled average density is always equal to 1 :

$$
\widetilde{n}_{\mathrm{ave}}=\left\langle\psi^{\dagger} \psi\right\rangle=1 .
$$

\section{B. Computational scheme}

To study the motion of vortices, we discretize Eq. (8) on a uniform spatial lattice and solve it as an initial value problem, i.e., knowing the state of the system at some initial moment, we calculate the state at later times. This requires imposing suitable initial and boundary conditions (see below). The lattice in general has different lattice spacings in the $x$ and $y$ directions. We used an operator-splitting algorithm with separate updates for the Laplacian and potential terms in (8). Updates corresponding to the Laplacian were done using the Crank-Nicholson scheme, which is unconditionally stable. The complete algorithm is unitary and second-order accurate in space and time.

\section{Boundary and initial conditions}

To avoid effects of the boundaries on the motion of the vortices, we use periodic boundary conditions in both directions. The initial states for the runs are created in the following way. We begin with the following field, containing a vortex and an antivortex (the presence of an antivortex is necessary to satisfy the boundary conditions):

$$
\psi(z)=\prod_{z_{+} \in\left[Z_{+}\right]} \frac{\left(z-z_{+}\right)}{\left|z-z_{+}\right|} \prod_{z_{-} \in\left[Z_{-}\right]} \frac{\left(z^{*}-z_{-}^{*}\right)}{\left|z-z_{-}\right|},
$$

where $Z_{+}$and $Z_{-}$are the desired (complex) positions of the vortex and antivortex, and $\left[Z_{ \pm}\right]$denotes the set of positions including $Z_{ \pm}$and a few mirror images with respect to the boundary. Then, evolving the system in the imaginary time, we cool the system down. Positions of the vortices during the cooling do not change, so we can place the vortices in convenient locations. To minimize effects of the vortexantivortex interaction, we place them half of the total lattice length apart. After that, we turn on a superflow and begin evolution in real time. A representative initial state, before and after the superflow was turned on, can be seen in Figs. 1 and 2.

\section{Velocity measurements}

The aim of the simulations is to observe the motion of vortices in the presence of superflow. The order parameter of the superfluid, being a complex scalar, can be written as

$$
\psi=\sqrt{n} e^{i \theta},
$$

where $n$ is the local density of the superfluid. Then, $2 \boldsymbol{\nabla} \theta$ is the local superfluid velocity. The average velocity of the superflow $\vec{U}$ is calculated as an average over the entire lattice. The velocity of a vortex $\vec{V}$, on the other hand, is obtained from direct tracking of the vortex position during the simulation. In a GI system, we expect vortices to move with the flow: $\vec{V}=\vec{U}$. If the invariance is broken, they may behave differently. To see the actual behavior, we break GI by solving the problem on increasingly coarser lattices.

\section{PHENOMENOLOGICAL MODEL}

While the vortex velocity can be measured directly in our simulations, converting these measurements into a value of the Magnus force requires a model of forces acting on the vortex. A fairly conventional model is available for an isotropic fluid (which we expect to apply also in the GI limit on 


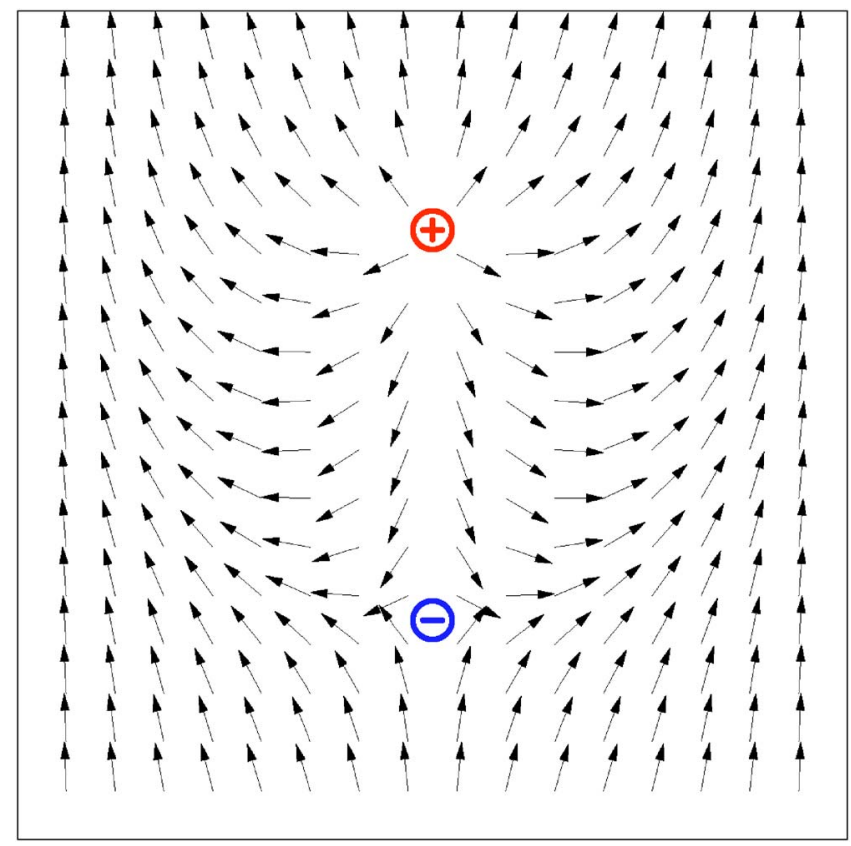

FIG. 1. (Color online) A representative initial state with two vortices of topological charge $1(+)$ and $-1(-)$ before superflow is turned on.

a lattice), but on coarse lattices modifications are needed. In this section, we review the conventional model, and then describe new effects introduced by the lattice.

\section{A. Magnus force in isotropic fluid}

The conventional (phenomenological) model includes three forces acting on a vortex (see Fig. 3). First, there is a

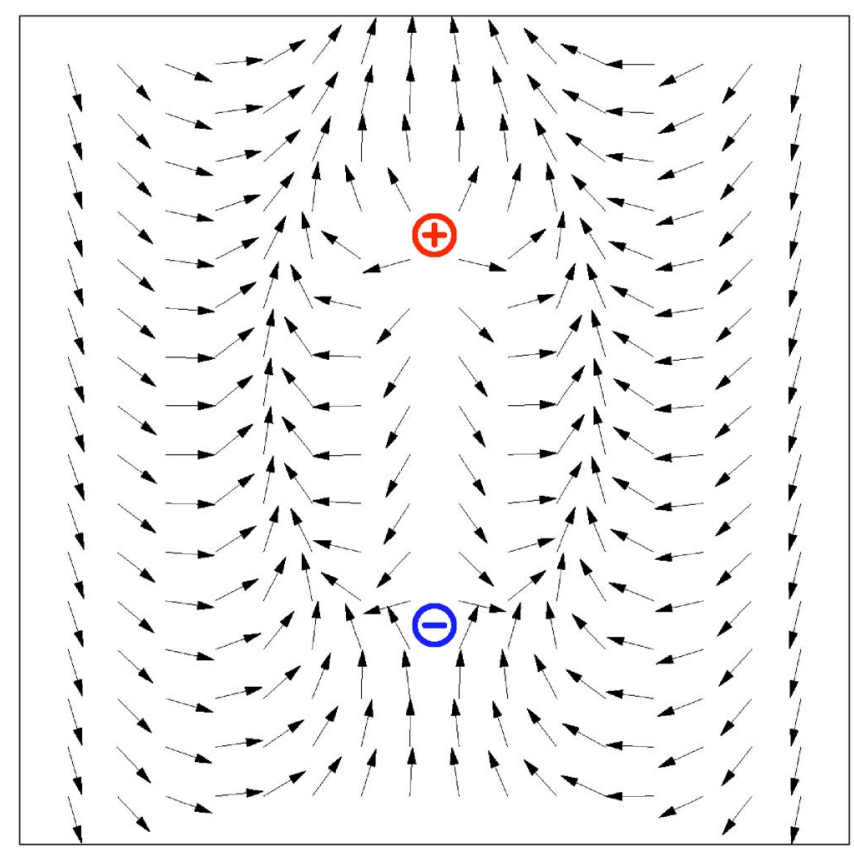

FIG. 2. (Color online) A representative initial state after superflow is turned on.

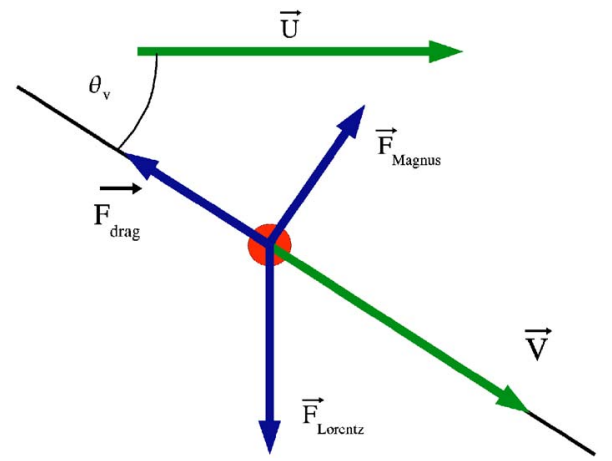

FIG. 3. (Color online) Diagram of forces acting on an (anti)vortex in an isotropic superfluid: $\vec{F}_{\text {Lorentz }}$ perpendicular to the superflow velocity $\vec{U}, \vec{F}_{\text {Magnus }}$ perpendicular to the vortex velocity $\vec{V}$, and $\vec{F}_{\text {drag }}$ opposite to $\vec{V}$. In dynamic equilibrium the sum of all forces vanishes, and the vortex moves at an angle $\theta_{v}=\pi / 2-\theta_{\text {Hall }}$ with respect to the superflow.

drag force $\vec{F}_{\text {drag }}=-\eta \vec{V}$, directed against the vortex velocity. It accounts for longitudinal momentum transfer from the vortex to the lattice and to excitations (quasiparticles). The latter channel is dissipative, i.e., the work done by the drag force goes into excitation of the quasiparticle subsystem. Close to the GI limit, we expect the classical momentum transfer to quasiparticles to be ineffective, and hence the drag to be small.

Second, there is an effective Magnus force $\vec{F}_{\text {Magnus }}=$ $-\sigma_{v} \hat{z} \times \vec{V}$, perpendicular to the vortex velocity.

Finally, there is a force perpendicular to the superflow velocity and accounting for the work done by vortices as they unwind the superflow. We refer to it as the Lorentz force, $\vec{F}_{\text {Lorentz }}=\sigma_{u} \hat{z} \times \vec{U}$. The coefficients $\eta, \sigma_{u}$, and $\sigma_{u}$ refer to unit inertial mass. We will never need to discuss the actual value of the vortex inertial mass in this paper.

So, the equation of motion for the vortex is

$\frac{\mathrm{d} \vec{V}}{\mathrm{~d} t}=\vec{F}_{\text {drag }}+\vec{F}_{\text {Magnus }}+\vec{F}_{\text {Lorentz }}=-\eta \vec{V}-\sigma_{v} \hat{z} \times \vec{V}+\sigma_{u} \hat{z} \times \vec{U}$

In complex notation, where we identify the $x$ direction with the real axis and the $y$ direction with the imaginary axis, the equation becomes

$$
\frac{\mathrm{d} V}{\mathrm{~d} t}=-\alpha V+\beta U
$$

where $\alpha=\eta+i \sigma_{v}$ and $\beta=i \sigma_{u}$.

The solution is easily found to be

$$
V=\frac{\beta}{\alpha} U+C \exp (-\alpha t),
$$

where $C$ is an integration constant. The exponential term is a transient that rapidly decays and turns out to be too small to be observed even at small times. At large times, it drops out altogether. Then, the solution becomes a motion with a constant velocity at an angle $\theta_{v}$, given by 


$$
\tan \theta_{v}=\arg \frac{\beta}{\alpha}=\eta / \sigma_{v},
$$

with respect to the supercurrent. The angle $\theta_{v}$ is related to the Hall angle $\theta_{\text {Hall }}$ frequently used in the literature by $\theta_{v}=\pi / 2$ $-\theta_{\text {Hall }}$.

The conclusion that vortices move in straight lines is well born out numerically. Notice that the steady velocity $V$ $=(\beta / \alpha) U$ depends only on the ratios $\sigma_{v} / \sigma_{u}$ and $\eta / \sigma_{u}$, characterizing the Magnus and drag forces. Measuring two components of the steady velocity, we obtain two equations for these two ratios, which can be solved with the result

$$
\begin{aligned}
& \frac{\sigma_{v}}{\sigma_{u}}=\frac{V_{x} U_{x}+V_{y} U_{y}}{V_{x}^{2}+V_{y}^{2}}, \\
& \frac{\eta}{\sigma_{u}}=\frac{V_{y} U_{x}-V_{x} U_{y}}{V_{x}^{2}+V_{y}^{2}} .
\end{aligned}
$$

\section{B. Magnus force on the lattice}

An immediate consequence of the above expressions is that changing the sign of vorticity, i.e., the signs of the coefficients $\sigma_{u}$ and $\sigma_{v}$, changes the motion of a vortex (which now becomes an antivortex) in such a way that the projection of the vortex velocity on the direction of $\vec{U}$ remains the same, while the orthogonal projection changes sign. In other words, a vortex and an antivortex move symmetrically with respect to the superflow. In general, for coarse lattices and superflow that is not parallel to one of the main periods of the lattice, we have found that the motion does not have that property. We interpret this as a result of anisotropy introduced by the lattice and by the superflow direction. To account for anisotropy, the net force in Eq. (12) needs to be replaced by

$$
\vec{F}=-\hat{M} \vec{V}-\sigma_{v} \hat{z} \times \vec{V}+\sigma_{u} \hat{z} \times \vec{U}
$$

where $\hat{M}$ is a symmetric matrix that can depend on the direction of $\vec{U}$. Such a matrix has three independent elements, which now replace the single drag coefficient of the isotropic model.

On the other hand, if the superflow velocity is along one of the main periods of the lattice, the vortex and antivortex do move symmetrically with respect to it. In this case, we can introduce the effective Magnus force and drag coefficients that are defined by Eqs. (16) and (17). In what follows, the superflow is always oriented along the $x$ direction, $\vec{U}$ $=\left(U_{x}, 0\right)$, and we present two types of results: one type is the ratios $V_{x} / U_{x}$ and $V_{y} / U_{x}$ themselves, which are directly measurable quantities, and the other is the effective force coefficients computed from Eqs. (16) and (17).

\section{RESULTS}

Simulations with different total lattice lengths have been carried out, with similar outcomes. The ratio of the vortex velocity to the superflow velocity for square lattices of the

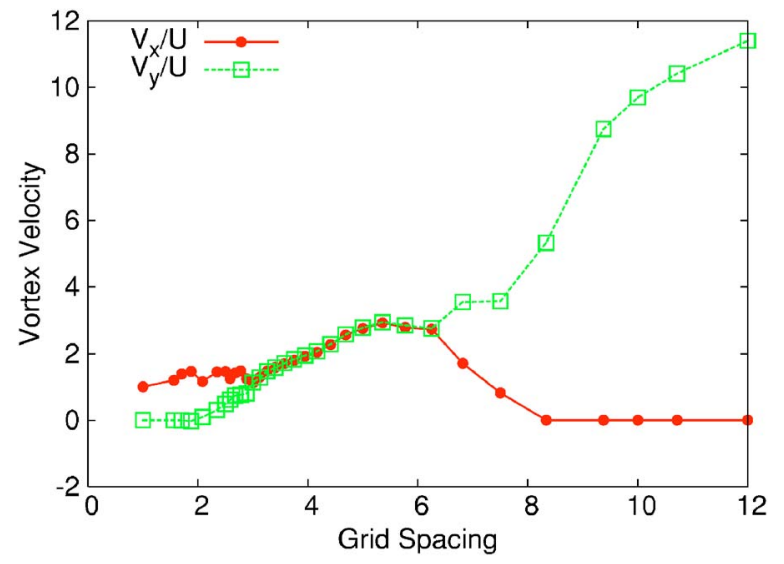

FIG. 4. (Color online) Longitudinal and transverse velocities of the vortex in units of the superflow velocity for different lattice spacings. The length of the lattice is 600 ; the superflow velocity is $\vec{U}=(0.07,0)$ in dimensionless units.

same length 600 (in the rescaled length units), constant superflow velocity $\vec{U}=(0.07,0)$, and different lattice spacings is shown in Fig. 4. Because the vortex has to overcome pinning in the lattice cells, it moves by detectable jumps. The data points were obtained by averaging the measured vortex velocities over long time intervals that begin some time after the start of the simulation.

We see that when the lattice spacing is close to 1 , in our dimensionless units, we obtain results expected for the GI limit: $V_{x} / U \approx 1$ and $V_{y} / U \approx 0$, i.e., $\vec{V} \approx \vec{U}$, which means that vortices go with the flow. For large spacings, i.e., in the discrete limit, the behavior changes radically. Now, $V_{x} / U$ $\approx 0$ and $V_{y} / U \gg 1$, i.e., vortices move perpendicular to the current. Between the two limits, there is an interesting regime when $V_{x}=V_{y}$, corresponding to motion with a Hall angle of $45^{\circ}$.

Let us see how these results are reflected in the parameters of our phenomenological model. As discussed in Sec. III B, we determine the effective force coefficients using

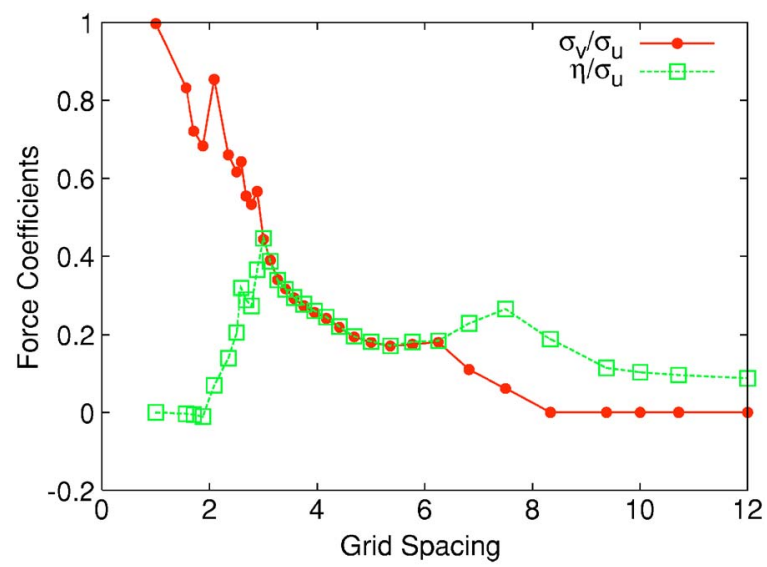

FIG. 5. (Color online) The ratios of the drag and Magnus force coefficients to the Lorentz force coefficient, as functions of the lattice spacing. The length of the lattice is 600; the superflow velocity is $\vec{U}=(0.07,0)$ in dimensionless units. 


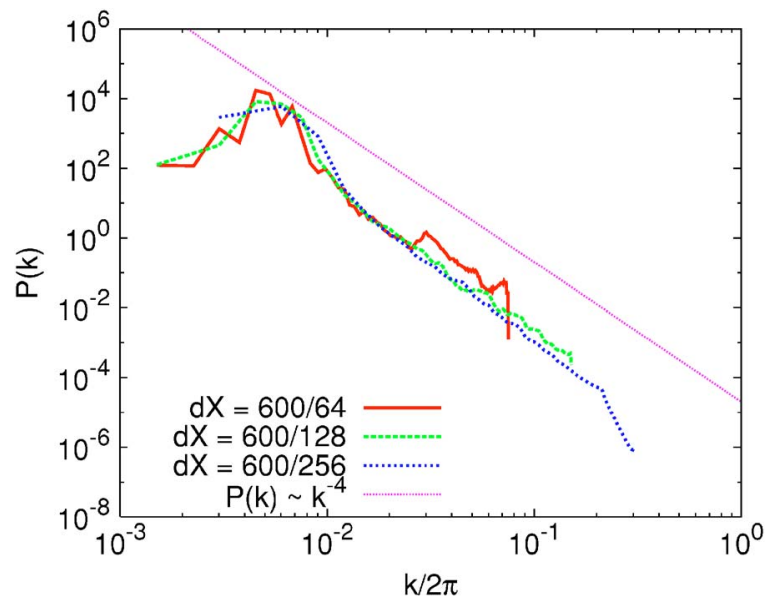

FIG. 6. (Color online) Power spectra of $\psi$ in the middle of the simulation for different values of the lattice spacing $d X$. The length of the lattice is 600 , and the lattice size (the number of lattice points) takes values $64^{2}, 128^{2}$, and $256^{2}$. The straight line is a $k^{-4}$ power law.

Eqs. (16) and (17). The result is shown in Fig. 5. In the quasi-continuous limit, we obtain $\sigma_{v} / \sigma_{u} \approx 1$ and $\eta / \sigma_{u} \ll 1$, meaning that the Magnus force coefficient is nearly equal to the Lorentz force coefficient, while the drag coefficient is very small in comparison.

In contrast, in the discrete limit, the Magnus force is nearly absent. This is our main result: we have observed a gradual vanishing of the Magnus force as we go from the continuous limit to the discrete one. We note that, while the Magnus force changes gradually, the drag coefficient has a rather sharp peak at the beginning of the region where $\sigma_{v}$ $\approx \eta$, i.e., when the vortex begins to move at $45^{\circ}$ to the flow.

The simplest intuitive picture that might account for the vanishing of the Magnus force is as follows. As the lattice spacing increases, the depletion of the density in the region of large phase gradients [the $\delta n(\nabla \theta)^{2}$ coupling discussed after Eq. (2)] becomes smaller, so that the system approaches the particle-hole-symmetric limit, in which the Magnus force is absent. ${ }^{10}$ A small remaining force, caused by deviations from this ideal limit, can be overcome by a force exerted by the lattice, resulting in zero net Magnus force.

To make sure that the variations of the observables with the lattice spacing are not due to variation in the population of short-wavelength modes, we check the power spectra. Figure 6 shows the power spectrum of the field $\psi$ in the middle of the simulation for three different values of the lattice spacing. From the plot, we infer that there are no major differences in the power spectra.

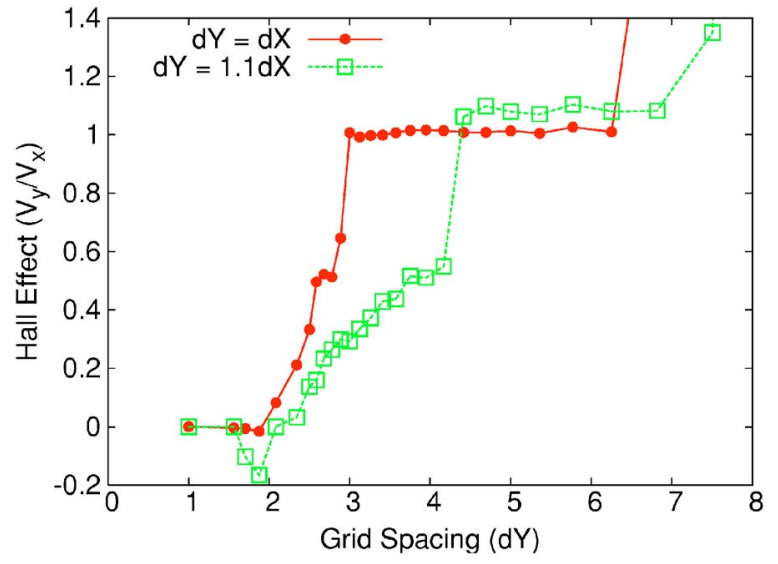

FIG. 7. (Color online) Hall effect $\left(V_{y} / V_{x}=\tan \theta_{v}\right)$ as a function of lattice spacing $\mathrm{d} Y$ for lattices with equal and unequal spacings in the $x$ and $y$ directions.

It is interesting to further explore the surprisingly broad intermediate range of lattice spacing where, as seen in Fig. 4, the Hall angle is close to $45^{\circ}$. We have found that this preferred direction is related to the geometry of the lattice, i.e., it remains along the diagonal of the unit cell even after we go from the square unit cell to a rectangular one (see Fig. 7).

\section{CONCLUSION}

The main result of the present paper is that in the classical limit (1), vortices in superfluids on coarse (but uniform) lattices, in the presence of a superflow parallel to one of the main periods, move perpendicular to the superflow. We interpret this result as a transition from the full Magnus force in the Galilean-invariant limit to vanishing effective Magnus force in the discrete limit, in agreement with the observed smallness of the Magnus force in JJAs. ${ }^{2}$ Our results are based on direct numerical simulations of the discrete superfluid (2) and do not assume a priori any symmetry that might prohibit the Magnus force in the discrete limit.

Another potential application of our results is vortex motion in cold atomic gases confined in optical lattices. If a sufficient degree of experimental control over parameters in either of these systems can be reached, intermediate points in the transition from the Galilean-invariant limit to the discrete limit may become observable. Notably, in our simulations these intermediate points include a somewhat counterintuitive regime where, for a broad range of lattice spacings, the average vortex velocity is along the diagonal of the unit cell.
${ }^{1}$ J. M. Harris, Y. F. Yan, O. K. C. Tsui, Y. Matsuda, and N. P. Ong, Phys. Rev. Lett. 73, 1711 (1994).

${ }^{2}$ For a review, see R. Fazio and H. van der Zant, Phys. Rep. 355, 235 (2001).

${ }^{3}$ N. B. Kopnin and V. E. Kravtsov, Zh. Eksp. Teor. Fiz. 71, 1644 (1976) [Sov. Phys. JETP 44, 861 (1976)].
${ }^{4}$ G. E. Volovik, Zh. Eksp. Teor. Fiz. 104, 3070 (1993) [JETP 77, 435 (1993)].

${ }^{5}$ A. van Otterlo, M. Feigel'man, V. Geshkenbein, and G. Blatter, Phys. Rev. Lett. 75, 3736 (1995).

${ }^{6}$ M. V. Feigel'man, V. B. Geshkenbein, A. I. Larkin, and V. M. Vinokur, Pis'ma Zh. Eksp. Teor. Fiz. 62, 811 (1995) [JETP Lett. 
62, 834 (1995)].

${ }^{7}$ M. Stone, Phys. Rev. B 54, 13222 (1996).

${ }^{8}$ G. E. Volovik, cond-mat/9707136.

${ }^{9}$ R. Fazio, A. van Otterlo, G. Schön, H.S.J. van der Zant, and J.E.
Mooij, Helv. Phys. Acta 65, 228 (1992).

${ }^{10}$ E. B. Sonin, Phys. Rev. B 55, 485 (1997).

${ }^{11}$ Yu. G. Makhlin and G. E. Volovik, Pis'ma Zh. Eksp. Teor. Fiz. 62, 923 (1995) [JETP Lett. 62, 941 (1995)]. 